International Journal of Modern Physics A

(C) World Scientific Publishing Company

\title{
BARYONIC DECAYS OF CHARMONIUM - A WINDOW ON INTERNAL BARYON STRUCTURE
}

\author{
A. Cieplý \\ Institute of Nuclear Physics, CZ-250 68 Řež, Czechia; \\ Institute of High Energy Physics, P.O.Box 918, Beijing 100049, China \\ cieply@ujf.cas.cz
}

\begin{abstract}
The baryonic decays of $J / \psi$ provide a new way to study the internal structure of baryons. A simple diquark model applied to the calculation of the $\bar{B} B$ decay cross-sections is compared with the ordinary constituent quark model. Various models also give different predictions for the rates involving the $N^{*}(1440)$ resonance in the final state.
\end{abstract}

Keywords: decays of $J / \psi$; hadron production; diquarks; Roper resonance

\section{Introduction}

In spite of a long history of baryon spectroscopy there are still many questions without clear answers. We know that the baryons are composed of three valence quarks, sea quarks and gluons but we are not certain if the valence quarks have constituent or current nature, if they cluster into diquarks or are well separated from each other etc. For a long time we have also missed direct sources of information on the properties of nucleon excitation states and could build our knowledge about them almost entirely on results from partial wave analysis of $\pi N$ scattering data. The situation is changing dramatically with new experimental data coming from facilities such as CEBAF at JLAB, ELSA in Bonn, GRAAL in Grenoble or from BEPC in Beijing. Especially the recent (and still not conclusively confirmed) discovery of pentaquark state is adding a flavour to the on-going discussion on the baryon and possibly multiquark internal structures.

A long-standing problem in $N^{*}$ physics is about the nature of the Roper resonance $N^{*}(1440)$ which is considered to be the first radial excitation state of the nucleon. However, various quark models have difficulty to explain its mass and electromagnetic coupling, so it was suggested that it may be a gluonic excitation of the nucleon, a hybrid baryon. The $J / \psi$ decays into baryon-antibaryon states discussed in the present contribution provide a novel way to probe the internal structure of baryons, their resonances and maybe the exotic states. 


\section{The models}

The $J / \psi$ decay cross sections for various $\bar{B} B$ final states were calculated in Refs 12 by using a simple quark model. The authors assumed that the decays proceed in two steps. First, the $\bar{c} c$ pair annihilates in three gluons and each gluon forms the quarkantiquark pair. Then, the three quarks and antiquarks combine to form the final state baryon and antibaryon. The lowest order of perturbative QCD and constituent quark model wave functions were used to calculate the relative branching ratios and the angular distributions of the specific final states.

More recently ${ }^{3}$ we used the diquark model to look into the possibility of forming the final state baryons from the $\bar{q} q$ (generated in $J / \psi$ decay) and diquarkantidiquark $\bar{D} D$ pairs (created as vacuum excitation). The model resembles the standard quark-pair-creation mode $\frac{4}{4}$ used extensively to describe the mechanism of hadronic decays. In this model, the transition matrix elements for charmonium

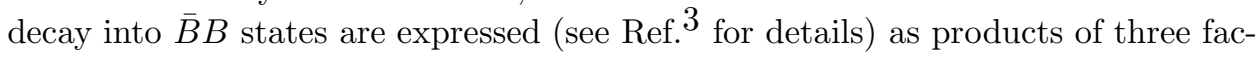
tors: the amplitude of creating a specific $\bar{q} q$ pair, the spin-flavour overlap amplitude following the standard SU(3) quark-diquark decomposition of the baryonic states, and the space integral involving the intrinsic wave functions of the baryon and the intermediate $\bar{q} q$ states.

Finally, we refer to the model suggested in Ref $\left[\frac{5}{5}\right.$ which uses the Roper resonance $N^{*}(1440)$ as a mixture of the standard three quark state $|q q q, 2 s\rangle$ and the gluon flavoured hybrid state $|q q q g\rangle$. In this model one of the three gluons created in the $\bar{c} c$ annihilation may become a constituent of the Roper resonance formed in the final state. At the same time one of the three $\bar{q} q$ pairs combining into the $\bar{B} B$ final state is created as vacuum excitation.



(a)

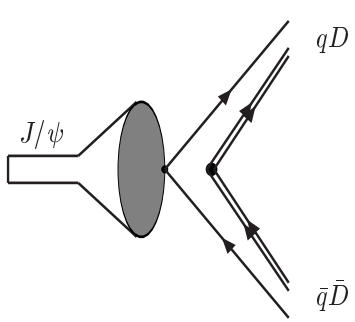

(b)

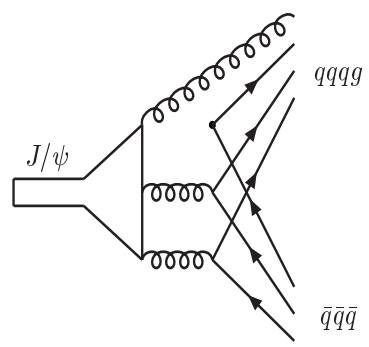

(c)

Fig. 1. A schematic illustration of the discussed models: (a) the standard $|q q q\rangle$ quark model, (b) the $|D q\rangle$ diquark model, (c) the hybrid $|q q q g\rangle$ model.

In the calculations simple gaussian distributions were used for the intrinsic baryon wave functions (and for the intermediate $\bar{q} q$ states appearing in the diquark model). Their harmonic oscillator parameters characterize the sizes of the relevant interaction and we varied them to fit the experimental data. Following Ref ${ }^{2]}$ both 
the nonrelativistic and relativistic treatments are considered in our calculations. In the later one the space integral includes the jacobian due to Lorentz transformation from the baryon CMS to the laboratory $(J / \psi$ at rest) system and the internal quark-diquark momenta are transformed appropriately as well23.

\section{Results and discussion}

In all models quoted above the decay cross-section for $J / \psi \longrightarrow \bar{B} B$ is constructed from the corresponding transition amplitudes and is expressed as

$$
\frac{d \Gamma(J / \psi \rightarrow \bar{B} B)}{d \Omega}=N_{\bar{B} B}\left(1+a_{B} \cos ^{2} \theta\right) .
$$

The constant $N_{\bar{B} B}$ is directly related to the given experimental branching ratio and we used $N_{\bar{p} p}$ to fix the overall normalization of the computed rates. The shape of the angular distribution, i.e. the value of $a_{B}$, was determined by the parameters $\alpha$ (for the intermediate $\bar{q} q$ state) and $\beta$ (for the baryon wave function) that characterize the intrinsic quark distributions.

The results of our calculation performed with the diquark mode 3 are presented in the Table 1 for both the nonrelativistic and the relativistic approaches. We show the relative decay rates $\Gamma_{\bar{B} B} / \Gamma_{\bar{p} p}$, where $\Gamma_{\bar{B} B}=4 \pi N_{\bar{B} B}\left(1+a_{B} / 3\right)$, and the angular distribution coefficients $a_{B}$ in comparison with the available experimental data. As the reliable data are limited only to the $\bar{p} p$ channel we further assumed $\alpha=\beta$ and made an one parameter fit to the measured value of $a_{p}$. The results shown in the Table 1 were obtained for $\alpha=\beta=0.4 \mathrm{GeV}$ and for $\alpha=\beta=0.22 \mathrm{GeV}$ in the nonrelativistic and the relativistic cases, respectively. Although the assumption of using the same size parameters for both the intermediate $\bar{q} q$ state and for the space distribution of baryon clusters may not be sound the fitted values compare well with those used in other quark models 28 .

Table 1. The computed characteristics $\Gamma_{\bar{B} B} / \Gamma_{\bar{p} p}$ and $a_{B}$ of the $J / \psi \rightarrow \bar{B} B$ decay rates.

\begin{tabular}{|c|cc|cc|cc|}
\hline \hline & \multicolumn{2}{|c|}{ nonrelativistic case } & \multicolumn{2}{|c|}{ relativistic case } & \multicolumn{2}{|c|}{ experiment } \\
$\bar{B} B$ state & $\Gamma_{\bar{B} B} / \Gamma_{\bar{p} p}$ & $a_{B}$ & $\Gamma_{\bar{B} B} / \Gamma_{\bar{p} p}$ & $a_{B}$ & $\Gamma_{\bar{B} B} / \Gamma_{\bar{p} p}$ & $a_{B}$ \\
\hline $\bar{p} p$ & 1.00 & 0.64 & 1.00 & 0.60 & 1.00 & $0.61(11) \sqrt{6}$ \\
$\bar{p} N^{*}$ & 0.90 & 0.53 & 0.76 & 0.68 & --- & --- \\
$\bar{N}^{*} N^{*}$ & 1.05 & 0.18 & 0.99 & 0.32 & --- & --- \\
$\bar{\Lambda} \Lambda$ & 0.56 & 0.50 & 0.54 & 0.54 & $0.61(9 \sqrt{7}$ & $0.62(22 \sqrt{6}$ \\
$\bar{\Sigma}^{0} \Sigma^{0}$ & 0.41 & 0.50 & 0.39 & 0.56 & $0.60(11) \sqrt{7}$ & $0.22(31 \sqrt{6}$ \\
\hline \hline
\end{tabular}

The results are compatible with available experimental data on $\bar{p} p, \bar{\Lambda} \Lambda$ and $\bar{\Sigma}^{0} \Sigma^{0}$ channels within two standard deviations. However the predicted results for the $\bar{p} N^{*}(1440)$ and $\bar{N}^{*} N^{*}$ are quite different from those given by the simple $|q q q\rangle$ quark mode ${ }^{2}$ and for the pure $|q q q g\rangle$ hybrid state ${ }^{5}$. The comparison of all three models is given in the Table 2 where our diquark model is labeled by $|D q\rangle$. The authors 
of Refs! 2 and $[5$ varied the model parameters to get the results within the limits shown in the Table 2] Future experimental results on the $N^{*}(1440)$ channels will be helpful for examining various model predictions and to improve our understanding of internal quark structure of these baryons. If the experimental $N^{*}$ production rates turn out to be much lower than the quark (and diquark) model predictions suggest a large component of the hybrid $|q q q g\rangle$ state could contribute to the $N^{*}$ internal structure. However, the simple models considered in this contribution cannot rule out conclusively some other mechanisms playing a role.

Table 2. The comparison of the results obtained for the $N^{*}(1440)$ channels within different models. Only the relativistic case is shown.

\begin{tabular}{|c|ccc|ccc|}
\hline \hline & \multicolumn{3}{|c|}{$\Gamma_{\bar{B} B} / \Gamma_{\bar{p} p}$} & \multicolumn{3}{c|}{$a_{B}$} \\
$\bar{B} B$ state & $|D q\rangle$ & $|q q q\rangle$ & $|q q q g\rangle$ & $|D q\rangle$ & $|q q q\rangle$ & $|q q q g\rangle$ \\
\hline $\bar{p} N^{*}$ & 0.76 & $2.0-4.5$ & $<0.02$ & 0.68 & $0.22-0.70$ & $0.42-0.57$ \\
$\bar{N}^{*} N^{*}$ & 0.99 & $3.2-22.0$ & $<0.002$ & 0.32 & $0.06-0.08$ & $-(0.1-0.9)$ \\
\hline \hline
\end{tabular}

\section{Conclusions}

Baryonic $J / \psi$ decays offer a novel tool to study the internal quark structure of baryons. Our diquark model calculation gives an alternative to more common quark models with different predictions for the formation rates of the $\bar{B} B$ final states. Specifically, the results obtained for the channels involving the $N^{*}(1440)$ Roper resonance depend significantly on the employed model. The calculations also show the importance of relativistic treatment for the distribution of baryon constituents. Future experimental data may provide a check on these different pictures for baryons.

\section{Acknowledgements}

This research was partially supported by the Grant Agency of the Czech Republic (grant No. 202/00/1667), CAS Knowledge Innovation Project (KJCX2-SW-N02) and the National Natural Science Foundation of China. The author acknowledges the hospitality of the IHEP in Beijing.

\section{References}

1. B.S. Zou et al., Euro. Phys. J. A 11, 341 (2001).

2. R.G.Ping, H.C.Chiang, and B.S.Zou, Phys. Rev. D66, 054020 (2002).

3. A. Cieplý and B.S. Zou, Czech. J. of Phys. 53, 639 (2003).

4. A. Le Yaouanc, L. Oliver, O. Pene, and J.C. Raynal, Phys. Rev. D8, 2223 (1973).

5. R.G.Ping, H.C.Chiang, and B.S.Zou, Nucl.Phys. A743, 149 (2004).

6. DM2 Collaboration, Nucl. Phys. B192, 653 (1987).

7. Particle Data Group, Phys. Rev. D66, 010001 (2002).

8. E.S. Ackleh, T. Barnes, and E.S. Swanson, Phys. Rev. D54, 6811 (1996). 